\title{
A Review on Various Optimized Image Watermarking Techniques
}

\author{
Vikas Sharma \\ M.Tech Scholar Department of CSE, \\ DAV institute of Engineering \& technology, \\ Jalandhar
}

\author{
P. S. Mann \\ Assistant Professor, Department of IT, \\ DAV institute of Engineering \& technology, \\ jalandhar
}

\begin{abstract}
This paper represents digital watermarking is a technique which allows an individual to add hidden copyright notices or other verification messages to digital audio, video, or image signals and documents. Two types of digital watermarks may be distinguished, depending upon if the watermark seems to be visible or even cannot be seen to the rare viewer. The complete goal of this paper is to explore the comparison of various techniques based on watermarking image and it also demonstrate that it provides the secured image watermarking with a decent capacity.
\end{abstract}

\section{Keywords}

Digital watermarking, Discrete Wavelet Transform (DWT).Watermarking techniques

\section{INTRODUCTION}

Watermarking plan top quality is usually made a decision putting on robustness, openness along with capacity. Openness usually means soon after installation of watermark the primary photograph really should not be distorted. Robustness pertains to attacks. If watermark treatment methods are tricky to diverse strikes such as turn, scaling, compression setting, noise next watermarking plan is usually robust. Total capacity usually means amount, which will are placed to pay for image. A lot more amount usually means it's possible to handle lots of files [2].Searching for watermark could possibly be obvious or invisible. An apparent watermark generally contains plainly obvious info or perhaps enterprise manufacturer exposing a usage from the image. Upon the next fretting hand, the invisibly watermarked impression appears just like the original. The existence of the invisible watermark can just simply be founded utilizing an suited watermark removals or acknowledgement algorithm. In this study most of us prohibit our own care about cannot be seen watermarks. A strong invisible watermarking approach, on the whole, consists of encoding approach and a decoding process. Watermarks involving varied quantity of coverage are extra with display mass media because amount of protection involving excellence, top quality, possession along with supplier [3].

\subsection{Features of digital watermarking}

Different top features of watermarking are as follows

\subsubsection{Robustness}

Robustness talks about how the watermark embedded in details offers the potential involving living adhering to many different control treatments and also attacks. Subsequently, the particular watermark should be solid intended for fundamental sign control function, geometric alteration and also damaging episode [4].

\subsubsection{Imperceptibility}

Watermark cannot be observed by simply observation or simply certainly not get noticed by simply individual scalp, just be found as a result of specific command or perhaps faithful circuits. It can be found by a state corporation only. Like watermarks are used by material or perhaps author accreditation and for stinking unauthorized copier.

\subsubsection{Safety}

The watermark practice is known as safeguarded, in the event the nuller can't remove the watermark without the need of total familiarity with embedding algorithm formula, detector in addition to arrangement involving watermark [5].Your watermark have to simply be readily available simply by licensed parties. That will need is considered as a burglar plus the watermark is mostly obtained simply by the application of cryptographic keys. Watermark data possesses the correct gauge to understand, the licensed men and women may possibly formally identify, find and even affect the watermark, and therefore control to obtain the aim involving hallmark protection.

\subsubsection{Verifiability}

Watermark ought to control to supply complete and trustworthy information for the possessing copyrightprotected information and facts products. The item may be used to discover if thez item will likely be attached and keep an eye on disperse involving the info staying attached, discover the particular authenticity, and purchase a grasp with against the law plagiarizing [6].

\subsubsection{Volume and knowledge payload}

Amount with the watermarking plan pertains to the best quantity of information which can be jammed in the duvet work. The quantity of watermark sections within the electronic mail in know-how payload and also the many regularity of knowledge payload in an image could be the watermark capacity. With regards to the plan a number of watermarking tactics need a knowledge payload going above 10,000 bits. Any watermark often have huge know-how volume level nevertheless small know-how payload.

\subsubsection{Computational charge}

In an effort to lessen computational fee, some sort of watermarking tactic has to be less complex. Watermarking tactics using big complex algorithms is going to requirement much more request and also consumer electronics resources and therefore bear much more computational cost. A Computational ease is normally decided on in resourcelimited ecosystem including cell devices.

\subsubsection{Watermark recognition stability}

In order to model highly effective watermarking in the trademark safety scenario, we could try a watermark in which 
has a pseudo-random binary schedule so that you can represent your identity of the trademark holder. The link amount involving the identity and also a properly found watermark is mostly superb established alongside the relationship amount involving the identity and also with little thought picked watermark. In cases like this your information associated with connection costs plotted from watermarks comes with a considerable leading with the adequately found watermark which fits on the trademark holder'utes identity. That is definitely watermark reputation result [7].

\subsubsection{Blind or non-blind recognition of watermark}

Some sort of watermarking method is supposedly shades, when it does not demand unique impression to get better the particular watermark in the watermarked image. Otherwise, a watermarking method is supposedly non-blind, when it will take unique impression pertaining to receiving the watermark in the watermarked image. The particular shades method is also referenced when oblivious. The particular non-blind watermarking solutions are generally a lot healthier as compared to shades watermarking solutions owing to option of unique take care of impression before detection. Nonetheless, shades or ignorant watermarking solutions are generally far more popular. The particular ignorant watermarking solutions reduce the over head of cost along with safe-keeping for saving unique photos [8].

\subsection{Discrete Wavelet Transform (DWT)}

The wavelet enhance possesses attained wide-spread recognition throughout indicator processing as well as picture compression. Lately a JPEG panel possesses released it's completely new picture programming standard, JPEG-2000, that is based on DWT. Wavelet enhance, breaks down a signal into a set of foundation functions. These kind of foundation capabilities are called wavelets. Wavelets will be obtained from just one magic size wavelet termed mum wavelet by way of dilations as well as shifting. The DWT continues to be announced because an extremely efficient and versatile method for sub band decomposition regarding signals. The 2D-DWT is usually nowadays proven for a crucial function throughout picture processing .It is usually multi-resolution examination and it breaks down pictures into wavelet coefficients as well as scaling function. Around Distinct Wavelet Convert, indicator power aims at to precise wavelet coefficients. That typical is useful for modifying images.

\section{RELATED WORK}

M. Ejima and A. Miyazaki, 2002[11] discussed Copyright laws safety involving electronic digital material gets to be a lot more critical prior to the creation of electronic digital technology. Nonetheless, it is sometimes complicated for people to help choose which strategy is preferable to others because the determine for evaluating the performance has not been set up yet. We all propose a way which evaluates this performance on the correlation-based watermarking method with the regularity domain. Piyu Tsai and $\mathrm{Yu}-\mathrm{Chen} \mathrm{Hu}$, 2005[12] proposed a verification from the items in transmission grayscale pictures. Throughout built, a watermark of merely one provided picture is made via the two rate area along with spatial domain. The trial and error success show that a consist of plan may distinguish suitable surgical procedures via harmful manipulations. Additionally, a placements involving expected altered locations is usually located properly along with recovered approximately.T. El
Areef, H. S. Heniedy and O. M. Ouda, 2006[13] investigates the problem of selecting the very best domain for embedding watermarks in digital images. The examined methods are selected to signify the different methods to embedding knowledge in spatial domain, distinct Fourier transform (DFT) domain, distinct cosine transform (DCT) domain, and distinct wavelet transform (DWT) domain. The calculations will also be plumped for to signify a range of computational difficulties and implementation structures. Masayoshi Nakamoto, Suguru Fujimoto, Akimitsu Doi and Takao Hinamoto,2008[14]Discussed pertaining to copyright laws defense, the part design is definitely embedded in discrete wavelet alter (DWT) site through a magic formula key sequence. The idea demonstrates the structure process on the key series in accordance with the genetic protocol (GA) so the robustness on the watermark is definitely strong. Eventually, many of us present the particular robustness in opposition to JPEG attack by way of laptop simulation. B. Jagadeesh, S. S. Kumar and K. R. Rajeswari, 2010 [15] discussed Digital camera Watermarking facts covering has grown to be a vital instrument regarding preserving digital illustrations or photos coming from theft, illegal copying and unlawful reproduction. A few digital watermarking algorithms were being proposed using spatial sector and transform domain. A transform sector may very well be DFT, DCT, DWT or perhaps SVD. Various digital watermarking algorithms using Inherited Algorithms can be bought in the particular literature. Shi et al., 2012 [16] has identified an RST invariant watermarking scheme applying DWT-SVD and logistic chart in which the watermark is a visually important grayscale logo. The outcomes demonstrate that scheme is able to endure a variety of geometric attacks and some common indicate method additive noise.Yang Qianli et al., 2012 [17] introduced an electronic watermarking algorithm with gray photograph centered on two dimensions which are distinct wavelet and cosine convert for guarding digital media trademark efficiently. Eventually, the key photograph is purchased by opposite convert of wavelet and cosine domain. Eventually, in this the watermarking is effective to the most used indicate processing practices, like JPEG compressing and noise. Zhu et al., 2013 [18] has described the particular electronic digital watermarking is a vital method of brand safety, that will breaks down RGB intended for colour photograph in addition to embeds the particular criteria of assorted watermarks in the grayscale photograph of 3rd r, G. The particular criteria has boosts into your market of watermark cornered in addition to eliminates the particular interference situation of embedding quite a few watermarks. Divecha and Jani, 2013 [19]defined automated picture watermarking is actually a design which has been designed to safeguarded automated substance from against the law use. Author organized the inclusion and examination of 2 numerous watermarking techniques determined by DCT-DWT-SVD. They are helpful to examine the success to get imperceptibility plus robustness by which maximum indication sound ratio plus normalized crosscorrelation boundaries are generally used. Ghaderi et al., 2013[20] investigated a tale semi-blind watermarking procedure for image hallmark protection, that's created in your

Training Wavelet Change (LWT) and it's created on Unique Price Decomposition (SVD). People used fractal decoding to develop a extremely stream-lined manifestation involving watermark image. tests claim that your watermark works from different violence, this sort of in terms of instance typical filter, Jpeg retention, spinning in addition to etc. 


\section{COMPARISON TABLE}

Table 1: Comparison of Various Techniques

\begin{tabular}{|c|c|c|c|c|c|}
\hline $\begin{array}{l}\text { Ref } \\
\text { No }\end{array}$ & Authors & Year & Technique & Features & Limitations \\
\hline [1] & Ansari ,et al & 2016 & Ant Bee Colony (ABC ) & $\begin{array}{l}\text { To find out the rightful } \\
\text { ownership, visible improvement } \\
\text { in performance. }\end{array}$ & $\begin{array}{l}\text { A Meta heuristic technique has } \\
\text { not been considered. }\end{array}$ \\
\hline [2] & C. N. Sujatha, et al & 2015 & MSE, PSNR and CF & $\begin{array}{l}\text { It illustrate the good } \\
\text { imperceptibility } \\
\text { and robustness }\end{array}$ & $\begin{array}{l}\text { The solution is not considered } \\
\text { to be efficient }\end{array}$ \\
\hline [3] & Bijoy Babu, et al & 2014 & DCT-SVD & $\begin{array}{l}\text { Producing good quality } \\
\text { watermarked images }\end{array}$ & $\begin{array}{l}\text { Because of some architectural } \\
\text { issues the solution is not } \\
\text { scalable and efficient. }\end{array}$ \\
\hline [4] & Tapan Jain, et al & 2014 & SVD and DWT & $\begin{array}{l}\text { The cover image is modified in } \\
\text { its singular values rather than on } \\
\text { the DWT sub bands }\end{array}$ & $\begin{array}{l}\text { Evolutionary technique has not } \\
\text { been considered. }\end{array}$ \\
\hline$[5]$ & Saini, et al & 2014 & $\begin{array}{l}\text { SVD, DWT and Back } \\
\text { propagation neural network }\end{array}$ & $\begin{array}{c}\text { Efficient hybrid watermarking } \\
\text { approach }\end{array}$ & $\begin{array}{l}\text { A Meta heuristic technique has } \\
\text { not been considered. }\end{array}$ \\
\hline [6] & Zebbiche, et al & 2014 & $\begin{array}{l}\text { Just perceptual weighting } \\
\text { (JPW) model }\end{array}$ & $\begin{array}{l}\text { Enhance the robustness of the } \\
\text { watermarking scheme }\end{array}$ & $\begin{array}{l}\text { But not tremendously increases } \\
\text { the field of vision regarding } \\
\text { beach haze image. }\end{array}$ \\
\hline [7] & Ghaderi,et al & 2013 & $\begin{array}{l}\text { LWT-SVD and fractal } \\
\text { images }\end{array}$ & $\begin{array}{l}\text { SVD is applied to sub-bands of } \\
\text { transformed image, and embed } \\
\text { the watermark by modifying the } \\
\text { singular values }\end{array}$ & $\begin{array}{l}\text { Because of some architectural } \\
\text { issues the solution is not } \\
\text { scalable and efficient. }\end{array}$ \\
\hline [8] & Divecha, et al & 2013 & DCT-DWT-SVD & $\begin{array}{l}\text { To enhance effectiveness of both } \\
\text { techniques for Imperceptibility } \\
\text { and robustness PSNR and NCC } \\
\text { parameters are used. }\end{array}$ & $\begin{array}{l}\text { An adaptive power control } \\
\text { method to improve the } \\
\text { network energy efficiency is } \\
\text { ignored }\end{array}$ \\
\hline [9] & Xiaohong Yu,,et al & 2013 & DWT-SVD & $\begin{array}{l}\text { Able to withstand a variety of } \\
\text { geometric attacks and some } \\
\text { common signal processing such } \\
\text { as additive noise. }\end{array}$ & $\begin{array}{l}\text { The solution is not efficient } \\
\text { and scalable }\end{array}$ \\
\hline [10] & Cai Yanhong., et al. & 2012 & JPW,DWT & $\begin{array}{l}\text { Producing good quality } \\
\text { watermarked images. }\end{array}$ & $\begin{array}{l}\text { Bounded into foreground } \\
\text { images but real time videos } \\
\text { requires more enhancement. }\end{array}$ \\
\hline
\end{tabular}

\section{CONCLUSION}

A digital watermarking is probably the strong implies to uncover the particular unauthorized usage of copyrighted graphics. So this paper shows about the comparison of various techniques based on the image watermarking which shows that DWT is employed on the image then singular components are also used for watermarks principle. But still there are some issues that have not considered the use of watermark scrambling as well as SWT has also been ignored.
So in near future we will evaluate using hybrid PSO and GA based technique on watermark images.

\section{REFERENCES}

[1] M. Ejima and A. Miyazaki, "On the evaluation of performance of digital watermarking in the frequency domain," Image Processing, 2001. Proceedings. 2001 International Conferenceon, Thessaloniki,2001,pp.546549vol.2. doi:10.1109/ICIP.2001.958549. 
[2] Shih-Wei Sun and Pao-Chi Chang, "Image watermarking system based on centroid projection," Information, Communications and Signal Processing, 2003 and Fourth Pacific Rim Conference on Multimedia. Proceedings of the 2003 Joint Conference of the Fourth International Conferenceon,2003,pp.1158-1162vol.2. doi:10.1109/ ICICS. 2003. 1292642

[3] Shanjun Zhang and K. Yoshino, "Embedding QR code watermark in divided wavelet domain," TENCON 2004. 2004 IEEE Region 10 Conference, 2004, pp. 287-290 Vol. 1 doi:10.1109/TENCON.2004.1414413

[4] X. Huang, Y. Luo, M. Tan and D. Lin, "A Image Digital Watermarking based on DWT in Invariant Wavelet Domain," Image and Graphics, 2007. ICIG 2007. Fourth International Conferenceon,Sichuan,2007,pp.329-336. doi: 10.1109/ICIG.2007.152

[5] H. q. Wang, F. Shang, Q. c. Ji and J. c. Hao, "Robust Digital Watermarking Adopting Barcode in Image," 2009 First International Conference on Information Science and Engineering, Nanjing, 2009, pp.660-662. doi:10.1109/ICISE.2009.1037

[6] Goel, Bhavna, and Charu Agarwal. "An optimized uncompressed video watermarking scheme based on SVD and DWT." In Contemporary Computing (IC3), 2013 Sixth International Conference on, pp. 307-312. IEEE, 2013.

[7] Zhao, Ke, and Jiang-feng $\mathrm{Xu}$. "A new semi-blind watermarking scheme based on DWT-subsampling." In Software Engineering and Service Science (ICSESS), 2013 4th IEEE International Conference on, pp. 623-626. IEEE, 2013.

[8] Raval, Keta, and S. Zafar. "Digital Watermarking with copyright authentication for image communication." In Intelligent Systems and Signal Processing (ISSP), 2013 International Conference on, pp. 111-116. IEEE, 2013.

[9] Hamid Shojanazeri, Wan Azizun Wan Adnan, Sharifah Mumtadzah Syed Ahmad "Video Watermarking Technique for Copyright Protection and Content Authentication," IEEE International Journal of Computer Information Systems and Industrial Management Applications, vol.5, pp. 652-660, 2013.

[10] Kakkirala, Krishna Rao, Srinivasa Rao Chalamala, and Jyoti Dhillon. "A robust image watermarking using DWT, SVD and Torus automorphism." In Computational Intelligence and Cybernetics (CYBERNETICSCOM), 2013 IEEE International Conference on, pp. 160-163. IEEE, 2013.

[11] M. Ejima and A. Miyazaki, "On the evaluation of performance of correlation-based watermarking techniques in the frequency domain," Image Processing. 2002. Proceedings. 2002 InternationalConferenceon,2002,pp.III-457-III460vol.3.doi: 10.1109 /ICIP .2002. 1039004

[12] Piyu Tsai and Yu-Chen Hu, "A Watermarking-Based Authentication with Malicious Detection and Recovery," 2005 5th International Conference on Information Communications \& Signal Processing, Bangkok, 2005, pp. 865-869. Doi: 10. 1109/ ICICS. 2005.1689172

[13] T. El Areef, H. S. Heniedy and O. M. Ouda, "Performance Evaluation of Image Watermarking
Techniques," 2006 ITI 4th International Conference on Information

CommunicationsTechnology,Cairo,2006,pp.1-1. doi:10.1109/ITICT.2006.358285

[14] Masayoshi Nakamoto, Suguru Fujimoto, Akimitsu Doi and Takao Hinamoto, "Genetic algorithm approach for wavelet-based image watermarking," 2008 9th International Conference on Signal Processing, Beijing, 2008, pp. 5-8. doi: 10.1109/ ICOSP. 2008. 4697055

[15] B. Jagadeesh, S. S. Kumar and K. R. Rajeswari, "Image Watermarking Scheme Using Singular Value Decomposition, Quantization and Genetic Algorithm," Signal Acquisition and Processing, 2010. ICSAP '10. International Conference on, Bangalore, 2010, pp. 120-124. doi:10.1109/ICSAP.2010.71

[16] Shi, Hailiang, Nan Wang, Zihui Wen, Yue Wang, Huiping Zhao, and Yanmin Yang. "An RST invariant image watermarking scheme using DWT-SVD." In Instrumentation \& Measurement, Sensor Network and Automation (IMSNA), 2012 International Symposium on, vol. 1, pp. 214-217. IEEE, 2012

[17] Qianli, Yang, and Cai Yanhong. "A digital image watermarking algorithm based on discrete wavele transform and Discrete Cosine Transform." In Information Technology in Medicine and Education (ITME), 2012 International Symposium on, vol. 2, pp. 1102-1105. IEEE, 2012.

[18] Zhu, Yong, Xiaohong Yu, and Xiaohuan Liu. "An image authentication technology based on digital watermarking." In Sensor Network Security Technology and Privacy Communication System (SNS \& PCS), 2013 International Conference on, pp. 179-183. IEEE, 2013.

[19] Divecha, Nidhi, and N. N. Jani. "Implementation and performance analysis of DCT-DWT-SVD based watermarking algorithms for color images." In Intelligent Systems and Signal Processing (ISSP), 2013 International Conference on, pp. 204-208. IEEE, 2013.

[20] Ghaderi, Kayvan, Fardin Akhlaghian, and Parham Moradi. "A new robust semi-blind digital image watermarking approach based on LWT-SVD and fractal

[21] Zebbiche, Khalil, and Fouad Khelifi. "Efficient waveletbased perceptual watermark masking for robust fingerprint image watermarking."2014 IET Image Processing 8, no. 1 (2014): 23-32.

[22] Saini, Hemraj. "Efficient hybrid watermarking approach by using SVD, DWT, and Back Propagation Neural Network." In Advance Computing Conference (IACC), 2014 IEEE International, pp. 985-990. IEEE, 2014.

[23] Sharma, Preeti, and Tapan Jain. "Robust digital watermarking for coloured images using SVD and DWT technique." In Advance Computing Conference (IACC), 2014 IEEE International, pp. 1024-1027. IEEE, 2014

[24] Varghese, Justin, Omer Bin Hussain, Bijoy Babu, Jamshid M. Basheer, Saudia Subash, Mohamed Ramadan Saadi, and Mohamed Samiulla Khan. "An efficient DCT-SVD based algorithm for digital image watermarking." In Security Technology (ICCST), 2014 International Carnahan Conference on, pp. 1-6. IEEE, 2014. 
[25] C. N. Sujatha, P. Satyanarayana. "An Improved Hybrid Color Image Watermarking under Various Attacks." In International Journal of Advanced Research in Computer and Communication Engineering(IJARCCE), Vol. 4, Issue 3, pp.339-343. March 2015.
[26] Ansari, Irshad Ahmad, Millie Pant, and Chang Wook Ahn. "ABC optimized secured image watermarking scheme to find out the rightful ownership." OptikInternational Journal for Light and Electron Optics 127.14 (2016): 5711-5721. 